\title{
miR-106b-5p promotes cell proliferation and cell cycle progression by directly targeting CDKN1A in osteosarcoma
}

\author{
CHUAN HE ${ }^{1,2^{*}}$, HONGWEI CHEN ${ }^{1,2^{*}}$, YAN LIU ${ }^{1}$, XIAOLIN LI ${ }^{1}$, \\ $\mathrm{CHAOJU}_{\text {ZHANG }}{ }^{1}$, QUNYAN QIN ${ }^{1}$ and QIXIONG PANG ${ }^{1}$ \\ ${ }^{1}$ Department of Orthopedics, Jingzhou Traditional Chinese Medicine Hospital; \\ ${ }^{2}$ Bone Injury Teaching and Research Office, The Third Clinical Medical School \\ of Yangtze University, Jingzhou, Hubei 434000, P.R. China
}

Received December 24, 2018; Accepted October 9, 2019

DOI: $10.3892 /$ etm.2020.8574

\begin{abstract}
MicroRNA (miR)-106b-5p has been reported to act as both an oncogene and tumor suppressor in several tumors. The aim of the present study was to investigate the biological function of miR-106b-5p in osteosarcoma (OS). miR-106b-5p expression was observed to be significantly increased in OS tissues and cell lines. MTT assay and flow cytometry analysis determined that miR-106b-5p inhibitor transfection suppressed OS cell proliferation and induced cell cycle G0/G1 phase arrest. Furthermore, bioinformatics analysis and a luciferase reporter assay demonstrated that cyclin-dependent kinase inhibitor 1A (CDKN1A) was a potential target of miR-106b-5p. p21 protein expression was found to be significantly increased by miR-106b-5p downregulation in OS cells. Further analysis demonstrated that CDKN1A was downregulated in OS tissues and was negatively correlated with miR-106b-5p expression. Furthermore, upregulation of CDKN1A expression mimicked, whilst CDKN1A knockdown reversed the suppressive effects of miR-106b-5p inhibitor on OS cell proliferation and cell cycle progression. In summary, the present data suggested that miR-106b-5p promotes cell proliferation and cell cycle progression by directly targeting CDKN1A in OS.
\end{abstract}

\section{Introduction}

Osteosarcoma (OS) is the most common form of primary bone malignancy that frequently affects children, adolescents and young adults, with $\sim 3,260$ cases in the United States reported in 2017 (1). Although progress has been made in the treatment

Correspondence to: Dr Qixiong Pang, Department of Orthopedics, Jingzhou Traditional Chinese Medicine Hospital, 172 Jiangjin East Road, Shashi, Jingzhou, Hubei 434000, P.R. China

E-mail: pang_qxiogugu@126.com

*Contributed equally

Key words: osteosarcoma, microRNA-106b-5p, cyclin dependent kinase inhibitor $1 \mathrm{~A}, \mathrm{G} 0 / \mathrm{G} 1$ phase of OS, including surgery and chemotherapy, the five-year survival rate remains relatively low in OS patients (2). Numerous studies indicate that epigenetic changes including non-coding RNA signatures, DNA methylation and histone modifications are present in all human malignancies and can facilitate cancer development and progression $(3,4)$.

MicroRNAs (miRNAs) have attracted major interest due to their ability to affect a wide range of fundamental biological processes such as proliferation, invasion, differentiation and apoptosis (5). Studies into OS-specific miRNAs have identified their potential in the prevention and management of OS $(6,7)$. Based on the latest version of miRBase, 3,700 miRNAs loci were annotated in human tissues (8). Bioinformatic predictions suggest that $30-60 \%$ of the protein-coding genes are regulated by miRNAs (9). MiRNAs cause mRNA degradation and post-transcriptional downregulation by binding to the 3'untranslated region (UTR) of their target genes (10). miR-106b-5p, a member of the miR-106b-25 cluster, is mapped to human chromosome $7 \mathrm{q} 21$ (Chr7q21) locus and has been reported to be amplified in several types of human malignancies, including renal cell carcinoma (11), hepatocellular carcinoma (12), glioma (13), melanoma (14) and non-small cell lung cancer (15). The deleterious effects of miRNA dysregulation on malignant behaviors appear to be strongly associated with tumorigenesis and cell cycle progression (12-15). However, current understanding regarding the role of miR-106b-5p in OS development and progression remains limited.

Cyclin-dependent kinase (CDK) represents a family of proline-directed serine/threonine kinases with important regulatory roles in modulating cell division in response to extrinsic and intrinsic signaling events (16). CDK inhibitor 1A (CDKN1A) is considered as a critical and universal CDK-interacting protein, which binds to CDKs and/or its subunits (17). There is clear evidence that CDKN1A is involved in cell cycle progression, proliferation, survival, motility and senescence $(18,19)$. CDKN1A expression has been previously found to be downregulated in numerous human malignancies, the restoration of which has been observed to attenuate metastasizes in vivo $(19,20)$. To date, a number of studies have identified that some miRNAs are associated with the expression of CDKN1A, including miR-93 (21), miR-130a (22), miR-519d (23) and miR-4295 (24). In OS, knockdown of 
miR-95-3p has been shown to inhibit cell growth by epigenetically regulating CDKN1A (25).

The purpose of the present study was to explore the biological role of miR-106b-5p in OS and identify the critical tumor-suppressed targets of miR-106b-5p. To the best of our knowledge, the current study first revealed that CDKN1A was a direct target of miR-106b-5p in OS, which will establish the miR-106b-5p/CDKN1A axis in the development and progression of OS.

\section{Materials and methods}

Patients and tumor specimens. A total of 18 pairs of fresh surgically resected OS tissue and adjacent bone tissue, $5 \mathrm{~cm}$ from the edge of tumor site, were obtained from OS patients (age range, 13-68 years; sex, 12 females and 6 males) after diagnosis by experienced pathologists between March 2015 and September 2017 at the Jingzhou Traditional Chinese Medicine Hospital (Hubei, China). All collected tissues were immediately frozen in liquid nitrogen. The present study was approved by the Ethics Committee of Jingzhou Traditional Chinese Medicine Hospital and all patients provided their written informed consent.

Cell culture. Human OS cell lines (Saos-2, MG-63, SW1353 and U2OS), osteoblast cell line hFOB 1.19 and embryonic kidney cell line 293T were purchased from the American Type Culture Collection. All cell lines were cultured in RPMI-1640 medium (Gibco; Thermo Fisher Scientific, Inc.) with 10\% fetal bovine serum (Gibco; Thermo Fisher Scientific, Inc.), $100 \mathrm{U} / \mathrm{ml}$ penicillin and streptomycin (Invitrogen; Thermo Fisher Scientific, Inc.). Samples were maintained in a humidified atmosphere containing $5 \% \mathrm{CO}_{2}$ at $37^{\circ} \mathrm{C}$.

Oligonucleotides and cell transfection. Oligonucleotides, including miR-106b-5p inhibitors (5'-ATCTGCACTGTC AGCACTTTA-3') and negative controls (miR-NC, 5'-TTC TCCGAACGTGTCACGT-3') were designed and synthesized by Shanghai GenePharma Co., Ltd. The open reading frame of CDKN1A, generated from RNA samples of Saos-2 cells (forward, 5'-CACCATGTCAGAACCGGCTGGGGATG-3'; reverse, 5'-TTAGGGCTTCCTCTTGGAGAAGATCAGC-3'), was inserted into the pcDNA3.1 expression vector to generate overexpressing recombinant vector pcDNA3.1-CDKN1A (Shanghai GenePharma Co., Ltd.). Small interfering (si)RNA for CDKN1A (si-CDKN1A) and its NC (si-NC) were synthesized by Shanghai GenePharma Co., Ltd. Saos-2 or U2OS cells $\left(1 \times 10^{4}\right.$ cells per well) were seeded into six-well plates and transfected with $50 \mathrm{nM}$ miRNA, 100 pmol siRNA and/or $4 \mu \mathrm{g}$ plasmid using Lipofectamine ${ }^{\circledR} 2000$ (Invitrogen; Thermo Fisher Scientific, Inc.) for $48 \mathrm{~h}$ according to the manufacturer's instructions.

Reverse transcription-quantitative PCR $(R T-q P C R)$ analysis. Total RNA was extracted with TRIzol ${ }^{\circledR}$ reagent (Invitrogen; Thermo Fisher Scientific, Inc.) according to the manufacturer's protocol. For miR-106b-5p detection, the temperature protocol for reverse transcription of miRNA was as follows: $37^{\circ} \mathrm{C}$ for $60 \mathrm{~min}, 95^{\circ} \mathrm{C}$ for $5 \mathrm{~min}$ and the samples were subsequently kept at $4^{\circ} \mathrm{C}$. RT-qPCR was performed in triplicate using a miRVana ${ }^{\mathrm{TM}}$ real-time RT-PCR microRNA detection kit (Thermo Fisher Scientific, Inc.) with U6 as an internal control. The thermocycling conditions were as follows: Initial denaturation of $95^{\circ} \mathrm{C}$ for $2 \mathrm{~min}$, followed by 40 cycles of $95^{\circ} \mathrm{C}$ for $10 \mathrm{sec}, 55^{\circ} \mathrm{C}$ for $30 \mathrm{sec}$ and $72^{\circ} \mathrm{C}$ for $30 \mathrm{sec}$. For CDKN1A detection, cDNA was synthesized using a PrimeScript ${ }^{\mathrm{TM}} \mathrm{RT}$ reagent kit (Takara Bio, Inc.). The temperature protocol for reverse transcription of RNA was as follows: $37^{\circ} \mathrm{C}$ for $15 \mathrm{~min}$ and $85^{\circ} \mathrm{C}$ for $5 \mathrm{sec}$. The expression of CDKN1A mRNA was determined using SYBR Premix Ex Taq ${ }^{\mathrm{TM}}$ II (Takara Bio, Inc.) using primers (forward, 5'-TCTGGGGTCTCACTTCTTGG-3' and reverse, 5'-ATGTGAGGAAGGCTCAGTGG-3') with GAPDH (forward, 5'-TGCACCACCAACTGCTTA-3' and reverse, 5'-GGATGCAGGGATGATGTTC-3') as an internal control. The thermocycling conditions were as follows: Initial denaturation at $95^{\circ} \mathrm{C}$ for $10 \mathrm{~min}$, followed by 40 cycles of $95^{\circ} \mathrm{C}$ for $10 \mathrm{sec}$ and $58^{\circ} \mathrm{C}$ for $60 \mathrm{sec}$. Relative expression levels were calculated using $2^{-\Delta \Delta \mathrm{Cq}}$ method (26).

Cell proliferation assay. Saos- 2 or U2OS cells were seeded in 96-well plates at a density of $4 \times 10^{3}$ cells per well and routinely cultured $\left(37^{\circ} \mathrm{C} ; 5 \% \mathrm{CO}_{2}\right)$ for 5 days. At the indicated time points, $20 \mu 1$ MTT reagent was added to each well (Sigma-Aldrich; Merck KGaA) and incubated for another $2 \mathrm{~h}$ at $37^{\circ} \mathrm{C}$. The blue formazan crystals in each well were subsequently dissolved by adding $150 \mu 1$ dimethylsulfoxide (Sigma-Aldrich; Merck KGaA). Cell proliferation was evaluated by recording the absorbance value at $595 \mathrm{~nm}$ using Model 680 microplate reader (Bio-Rad Laboratories, Inc.).

Cell cycle analysis. Flow cytometry analysis was performed to determine cell cycle distribution in OS cells. Saos-2 or U2OS cells at a density of $4 \times 10^{5}$ cells per reaction, were washed with PBS and fixed with pre-cooled $70 \%$ ethanol at $4^{\circ} \mathrm{C}$ for $30 \mathrm{~min}$. Following centrifugation $\left(450 \mathrm{x}\right.$ g at $4^{\circ} \mathrm{C}$ and $\left.5 \mathrm{~min}\right)$ and washing with PBS, cells were stained with $500 \mu 11 \%$ propidium iodide (PI; Thermo Fisher Scientific, Inc.), followed by DNA content analyses using BD FACScan ${ }^{\mathrm{TM}}$ flow cytometer equipped with CellQuest Pro 4.0.2 software (BD Biosciences).

Luciferase reporter assay. The putative target genes of miR-106b-5p were predicted using public available algorithms, including PicTar (https://pictar.mdc-berlin.de), TargetScan (http://targetscan.org) and miRDB (http://www. mirdb.org). The predicted miR-106b-5p binding sites in the wild-type (WT) 3'UTR of CDKN1A and the corresponding mutant type (MUT) miR-106b-5p binding sites were cloned into a pGL3 vector (Promega Corporation). For the luciferase reporter assay, $293 \mathrm{~T}$ cells $\left(1 \times 10^{5}\right.$ cells/well $)$ were seeded in 96-well plates and co-transfected with $300 \mathrm{ng}$ WT-CDKN1A or MUT-CDKN1A, and $100 \mathrm{nM}$ of miR-106b-5p inhibitor or miR-NC using Lipofectamine ${ }^{\circledR} 2000$ (Invitrogen; Thermo Fisher Scientific, Inc.) in accordance with the manufacturer's protocol. After $48 \mathrm{~h}$ transfection, the relative luciferase activities were measured using the dual-luciferase reporter assay system (Promega Corporation). Renilla luciferase activity was used for normalization.

Western blot analysis. Total protein was extracted using the RIPA protein extraction reagent (Beyotime Institute of 
A

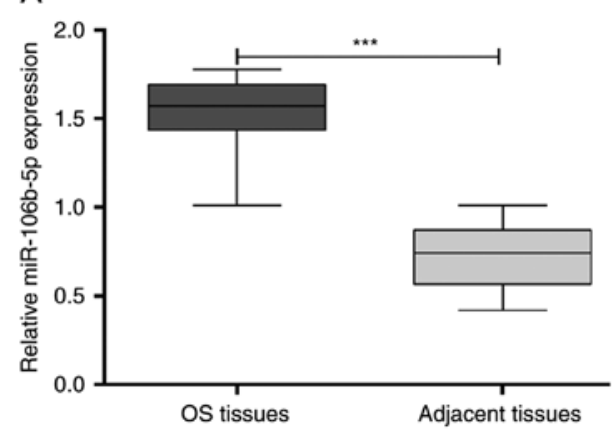

B

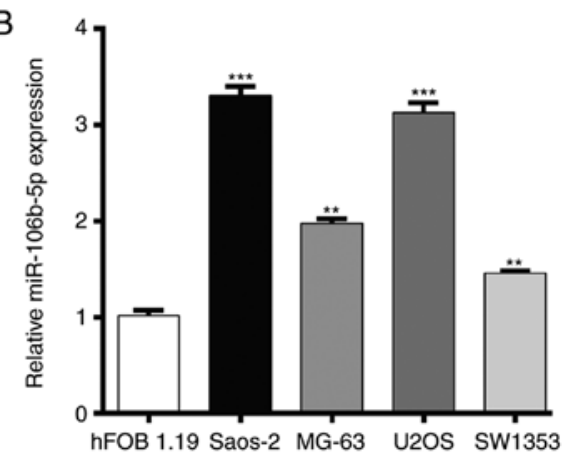

Figure 1. Expression of miR-106b-5p in OS clinical samples and cell lines. (A) Expression of miR-106b-5p in 18 pairs of human OS tissue and adjacent tissue, as determined via reverse transcription-quantitative PCR. ${ }^{* * *} \mathrm{P}<0.001$ as indicated. (B) Expression of miR-106b-5p in OS cells lines (MG-63, U2OS, SW1353 and Saos-2) and an osteoblast cell line hFOB1.19 is presented. Data are represented as the mean \pm standard deviation. ${ }^{* * *} \mathrm{P}<0.01$ and ${ }^{* * * *} \mathrm{P}<0.001 \mathrm{vs}$. hFOB1.19 cell line. miR, microRNA; OS, osteosarcoma.

Biotechnology) and the protein concentration was determined using the bicinchoninic acid Protein Assay kit (Beyotime Institute of Biotechnology). Approximately $30 \mu \mathrm{g}$ protein was separated by $10 \%$ SDS-PAGE and then transferred to nitrocellulose membranes (Bio-Rad Laboratories, Inc.). The membranes were blocked in 5\% non-fat milk containing $0.1 \%$ Tween-20 (TBST) for $2 \mathrm{~h}$ at room temperature and then probed with primary antibodies against p21 (1:1,000; cat. no. \#2947; Cell Signaling Technology, Inc.) and GAPDH (1:5,000; cat. no. \#2118; Cell Signaling Technology, Inc.) overnight at $4^{\circ} \mathrm{C}$. After washing with PBS, the membranes were incubated with a horseradish peroxidase-conjugated secondary antibody (1:20,000; cat. nos. ab205718; Abcam) for $2 \mathrm{~h}$ at room temperature. Protein bands were visualized with enhanced chemiluminescence reagents (Pierce Biotechnology; Thermo Fisher Scientific, Inc.) following the manufacturer's protocol.

Statistical analysis. All experiments were independently performed in triplicate. The data were analyzed with SPSS 19.0 software (SPSS, Inc.) and expressed as the mean \pm standard deviation. Differences between two groups were assessed using Student's t-test. Differences amongst multiple groups were evaluated using one-way ANOVA followed by Tukey's post-hoc test. Correlations between the expression of miR-106b-5p and the expression of CDKN1A were analyzed by Spearman's rank correlation. $\mathrm{P}<0.05$ was considered to indicate statistically significant difference.

\section{Results}

miR-106b-5p is highly expressed in OS tissues and cells. RT-qPCR was used to detect the expression of miR-106b-5p in tumor tissue and adjacent tissue derived from OS patients. As presented in Fig. 1A, miR-106b-5p was significantly upregulated in OS tissue compared with adjacent tissue $(\mathrm{P}<0.001)$. In addition, the expression of miR-106b-5p in several human OS cells lines including MG-63, U2OS, SW1353 and Saos-2 was also analyzed. As presented in Fig. 1B, miR-106b-5p expression was significantly higher in OS cell lines compared with the osteoblast cell line hFOB1.19 $(\mathrm{P}<0.01$ and $\mathrm{P}<0.001)$. These results suggested that increased miR-106b-5p may serve an important role in OS.
Downregulation of miR-106b-5p inhibits OS cell proliferation and cell cycle progression. Saos-2 and U2OS cells demonstrated relatively higher levels of miR-106b-5p and were therefore selected for miR-106b-5p inhibitor transfection to knockdown miR-106b-5p. RT-qPCR was utilized to validate transfection efficiency. The results demonstrated that transfection with the miR-106b-5p inhibitor significantly reduced miR-106b-5p levels in Saos-2 and U2OS cells compared with the miR-NC group (Fig. 2A; $\mathrm{P}<0.001$ ). The results of the MTT assay indicated that miR-106b-5p inhibition significantly suppressed the proliferation of Saos- 2 and U2OS cells at day 3, 4 and 5 (Fig. 2B; P $<0.001$ ). As cell proliferation may be regulated by cell cycle progression, flow cytometry analysis was performed to examine the cell cycle distribution in Saos-2 and U2OS cells. As presented in Fig. 2C, downregulation of miR-106b-5p caused an increase in the cell population in the $\mathrm{G} 0 / \mathrm{G} 1$ phase $(\mathrm{P}<0.001)$. Accordingly, the cell population in the $\mathrm{S}$ and $\mathrm{G} 2 / \mathrm{M}$ phase was reduced in Saos $-2(\mathrm{P}<0.01$ and $\mathrm{P}<0.001)$ and $\mathrm{U} 2 \mathrm{OS}$ cells $(\mathrm{P}<0.01$ and $\mathrm{P}<0.001)$. These results indicated that miR-106b-5p accelerated cell proliferation which may be closely associated with cell cycle progression in OS cells.

miR-106b-5p negatively regulates CDKN1A by binding to its 3'UTR. Bioinformatics analysis was performed to predict the potential target genes of miR-106b-5p involved in cell cycle regulation. Among the predicted targeted genes, three miR-106b-5p targets associated with cell cycle regulation, including CDC37L1, CDKN1A and CCNG2 were selected as potential target genes of miR-106b-5p. Luciferase reporter assay showed that there was no significant interaction between miR-106b-5p and the 3'UTRs of CCNG2 and CDC37L1 (Fig. S1). It was determined that CDKN1A, a gene associated with G1-S transition, was a putative target gene of miR-106b-5p (Fig. 3A). As expected, the luciferase activity of the miR-106b-5p inhibitor + WT-CDKN1A was significantly increased compared with the miR-106b-5p inhibitor + MUT-CDK1A in 293T cells (Fig. 3B). In addition, to further confirm whether CDKN1A was regulated by miR-106b-5p, the expression of CDKN1A in transfected Saos-2 and U2OS cells was examined using RT-qPCR and western blotting. The results demonstrated that miR-106b-5p downregulation significantly upregulated the expression of CDKN1A mRNA (Fig. 3C; $\mathrm{P}<0.01$ ) and protein levels (Fig. 3D and E) in both Saos-2 and 
A

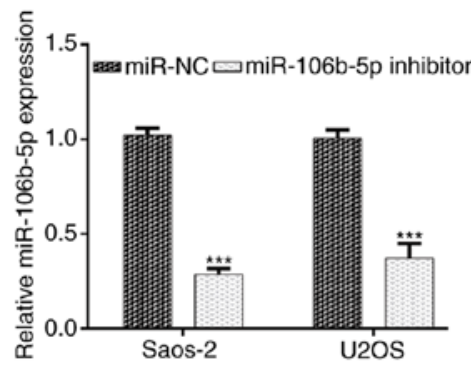

B

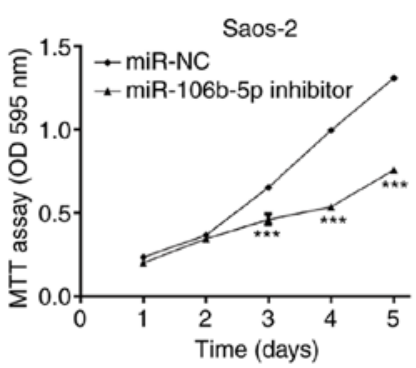

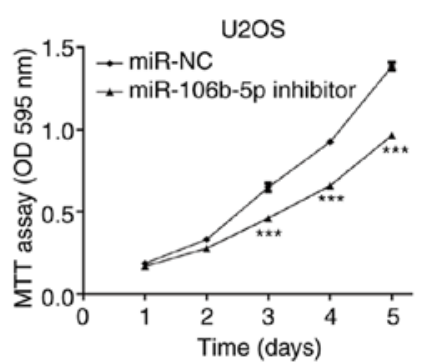

C
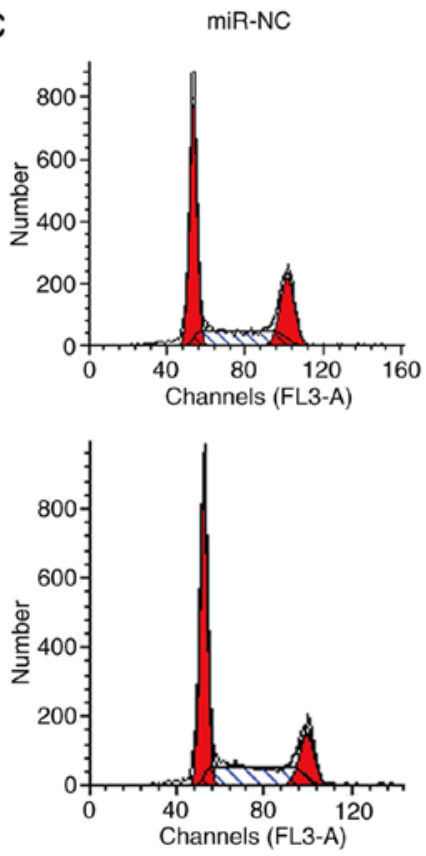

miR-106b-5p inhibitor
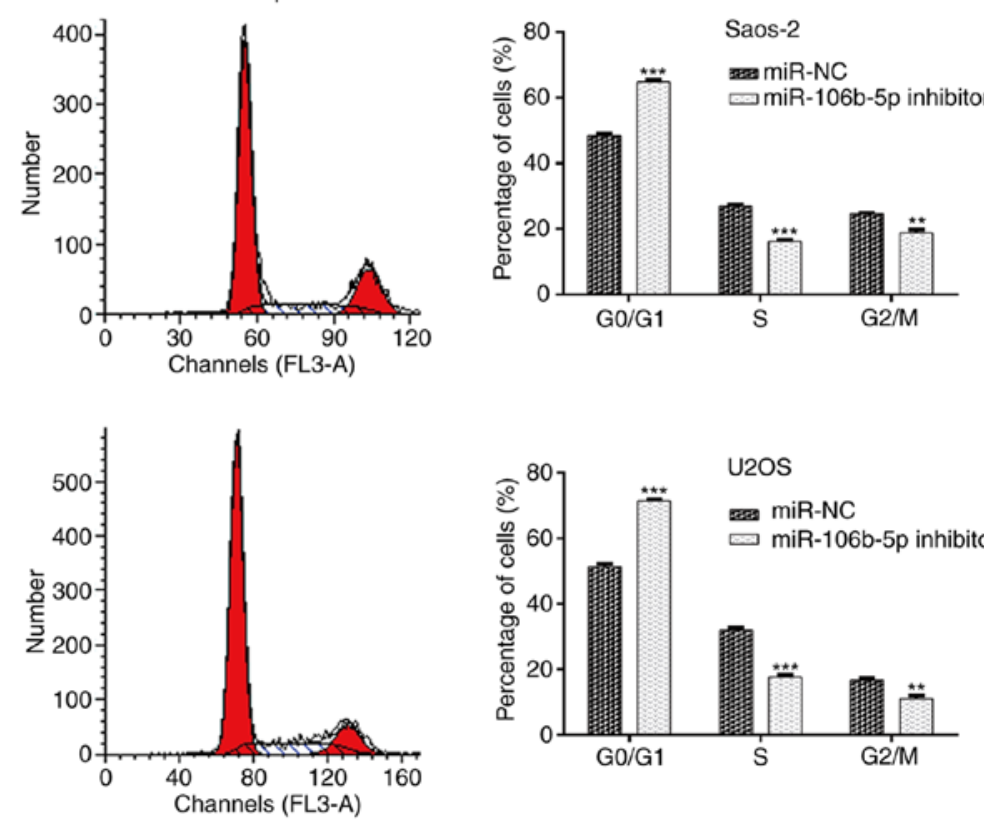

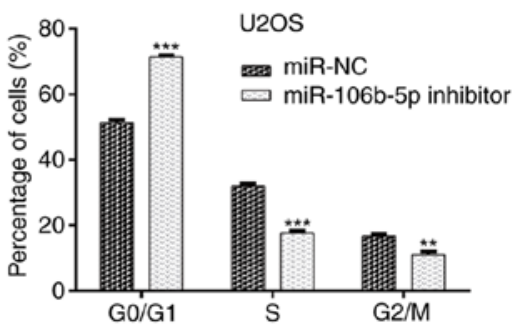

Figure 2. Downregulation of miR-106b-5p inhibited the proliferation and cell cycle progression of OS cells. (A) Saos-2 and U2OS cells transfected with the miR-NC or miR-106b-5p inhibitor were analyzed for miR-106b-5p expression via reverse transcription-quantitative PCR. (B) An MTT assay was performed to examine cell proliferation following transfection with the miR-NC or miR-106b-5p inhibitor. (C) Flow cytometry was performed to examine the cell cycle distribution of Saos-2 and U2OS cells following transfection with the miR-NC or miR-106b-5p inhibitor. Data are presented as the mean \pm standard deviation. ${ }^{* *} \mathrm{P}<0.01$ and ${ }^{* * *} \mathrm{P}<0.001$ vs. NC group. miR, microRNA; OS, osteosarcoma; NC, negative control; OD, optical density.

U2OS cells. Furthermore, the expression of CDKN1A in OS tissues was determined. As depicted in Fig. 3F, CDKN1A had a significantly lower expression in OS tissues compared with adjacent tissues $(\mathrm{P}<0.001)$. Through a two-tailed Pearson's correlation analysis, it was determined that the expression of miR-106b-5p was inversely correlated with CDKN1A expression in OS tissues (Fig. 3G; $\mathrm{P}=0.0057$ ). Taken together, the results suggested that CDKN1A was negatively regulated by miR-106b-5p in OS.

Overexpressed CDKNIA imitates the suppressive effects of miR-106b-5p inhibitor transfection on OS cells. To illustrate whether CDKN1A acts as a downstream effector in miR-106b-5p-mediated OS cell proliferation and cell cycle progression, gain-of-function assays were performed in Saos-2 cells by transfecting pcDNA3.1-CDKN1A. Following transfection, the mRNA (Fig. 4A; P<0.001) and protein (Fig. 4B) levels of CDKN1A were markedly increased in the pcDNA3.1-CDKN1A group compared with the control group in Saos-2 cells. The MTT assay further indicated that overexpression of CDKN1A suppressed the proliferation of Saos-2 cells, which was identical to the effect exerted by miR-106b-5p knockdown (Fig. 4C; $\mathrm{P}<0.001$ ). In addition, flow cytometry demonstrated that CDKN1A upregulation induced cell cycle arrest at the G0/G1 stage in Saos-2 cells, similar to the effect of miR-106b-5p knockdown (Fig. 4D; P<0.001). Collectively, these results suggested that CDKN1A might be a direct effector involved in the miR-106b-5p-mediated cell proliferation in OS.

CDKN1A knockdown abolishes the suppressive effects of miR-106b-5p inhibitor transfection on OS cells. To further confirm whether miR-106b-5p-mediated cell proliferation and cell cycle progression was dependent on its capacity to modulate CDKN1A expression, rescue experiments were performed by transfecting si-CDKN1A plasmid into Saos-2 cells treated with miR-106b-5p inhibitor. The expression of CDKN1A in Saos-2 cells was detected and it was determined that si-CDKN1A transfection markedly attenuated the increased CDKN1A mRNA $(\mathrm{P}<0.01$; Fig. 5A) and protein (Fig. 5B) expression. As expected, the decreased cell proliferation and induced cell cycle G0/G1 phase arrest by miR-106b-5p inhibitor were partially abolished by CDKN1A knockdown in Saos-2 cells, as determined by an MTT assay $(\mathrm{P}<0.01$ and $\mathrm{P}<0.001$; Fig. 5C) and flow cytometry analysis 


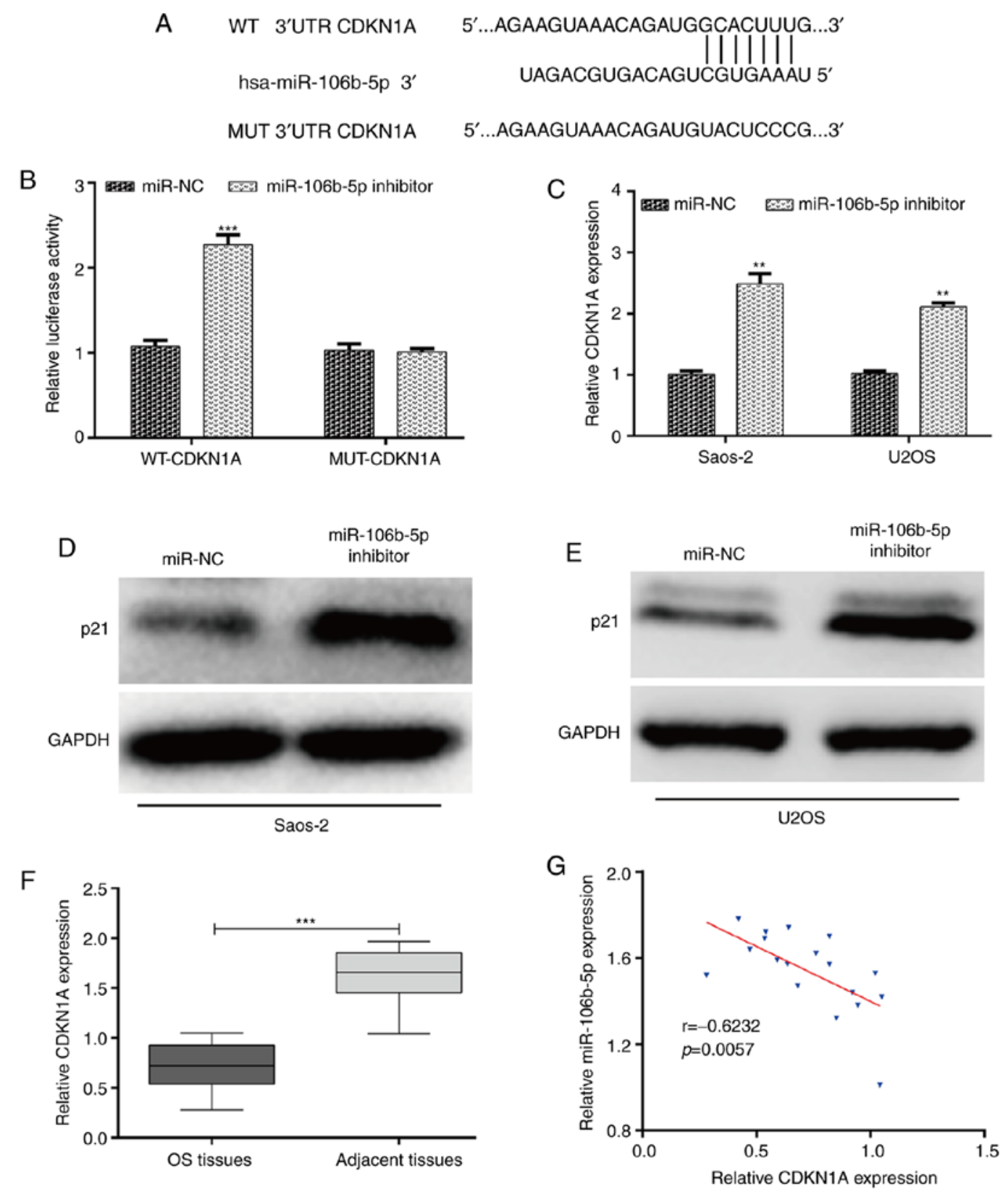

Figure 3. CDKN1A is a target gene of miR-106b-5p. (A) Binding sites between WT and MUT CDKN1A 3'-UTR and miR-106b-5p. (B) Luciferase reporter assays were performed on 293T cells transfected with WT or MUT CDKN1A 3'-UTR vectors and miR-106b-5p inhibitor or miR-NC. (C) RT-qPCR analysis of CDKN1A mRNA levels in Saos-2 and U2OS cells transfected with the miR-106b-5p inhibitor or miR-NC. (D) Western blot analysis of p21 protein level in Saos-2 and (E) U2OS cells transfected with miR-106b-5p inhibitor or miR-NC. (F) mRNA expression of CDKN1A relative to GAPDH in human OS tissues and adjacent tissues, as detected via RT-qPCR. ${ }^{* * *} \mathrm{P}<0.001$ as indicated. (G) A Negative correlation between miR-106b-5p and CDKN1A expression was identified in OS tissues. Data are presented as the mean \pm standard deviation. ${ }^{* *} \mathrm{P}<0.01$ and ${ }^{* * * *} \mathrm{P}<0.001$ vs. NC group. CDKN1A, cyclin-dependent kinase inhibitor 1A; miR, microRNA; WT, wild-type; MUT, mutant; UTR, untranslated region; NC, negative control; RT-qPCR, reverse transcription-quantitative; OS, osteosarcoma.

( $\mathrm{P}<0.001$; Fig. 5D). These results further demonstrated that CDKN1A might be a key regulator of miR-106b-5p-mediated cell proliferation and cell cycle progression in OS cells.

\section{Discussion}

Changes in miR-106b-5p levels are correlated with and facilitate cancer tumorigenesis. Some examples include the amplification of miR-106b-5p triggering stem cell-like properties of hepatocellular carcinoma cells (12), miR-106b-5p conferring proliferative advantage and inhibiting apoptosis in non-small cell lung cancer and overexpression of miR-106b-5p attenuating invasion and metastasis in colorectal cancer (27). By contrast, miR-106b-5p is reported to inhibit metastasis in colorectal cancer (27) and is associated with better survival in bladder cancer (28). However, whether miR-106-5p functions as an oncogene or tumor suppressor in OS still remains unclear. The present study assessed the differential expression of miR-106b-5p in OS clinical tissue and adjacent tissue, as well as in OS cell lines and a normal osteoblast cell line (hFOB1.19). The present results demonstrated that miR-106b-5p was overexpressed in OS tissues and cell lines using RT-qPCR. 
A

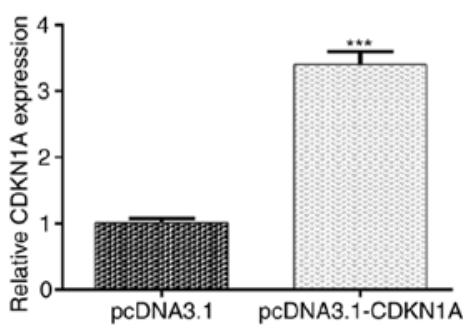

D

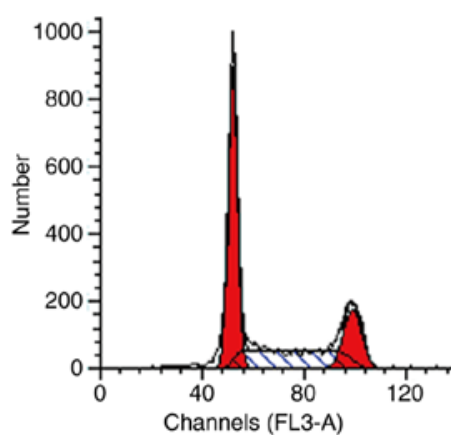

B

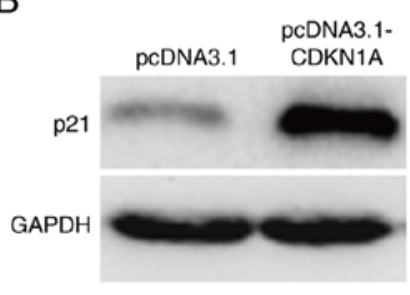

pcDNA3.1-CDKN1A

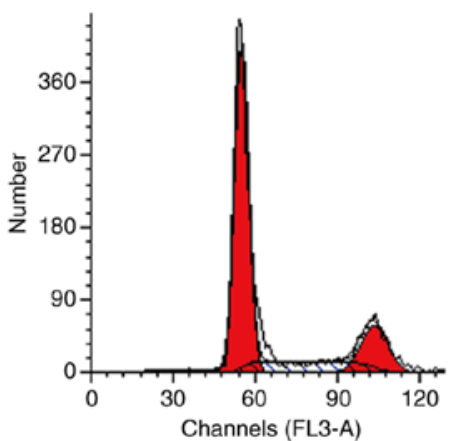

C
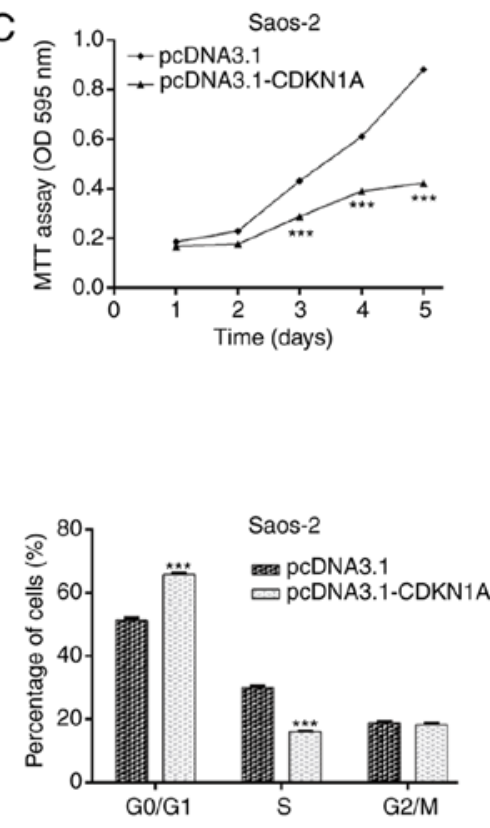

Figure 4. CDKN1A overexpression inhibits the proliferation and cell cycle progression of OS cells. (A) Reverse transcription-quantitative PCR and (B) western blotting were performed to examine the expression of CDKN1A in Saos-2 cells transfected with the blank pcDNA3.1 vector or pcDNA3.1-CDKN1A open reading frame plasmid. (C) An MTT assay was performed to examine cell proliferation and (D) flow cytometry was used to examine the cell cycle distribution. Data are presented as the mean \pm standard deviation. ${ }^{* * *} \mathrm{P}<0.001$ vs. pcDNA 3.1 group. CDKN1A, cyclin-dependent kinase inhibitor $1 \mathrm{~A}$; OS, osteosarcoma.
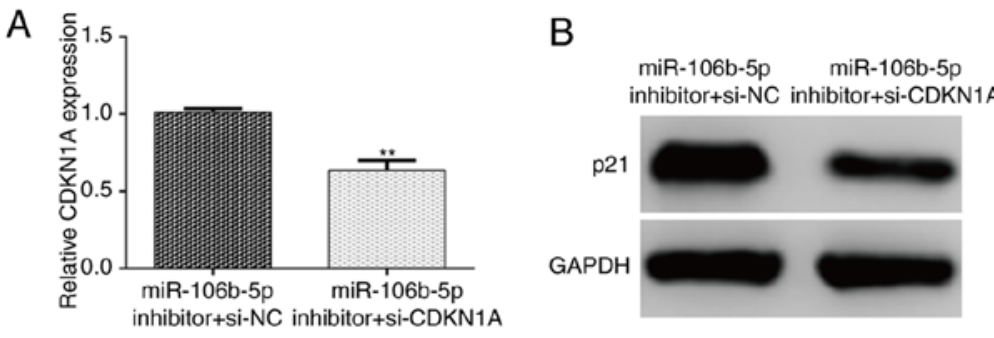

C

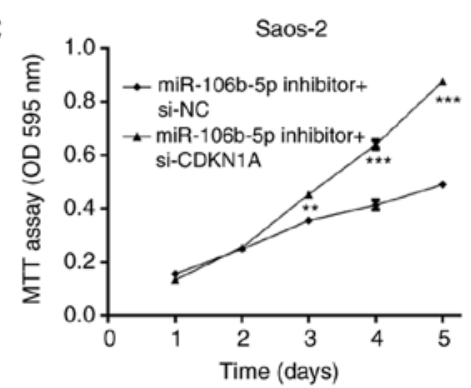

D

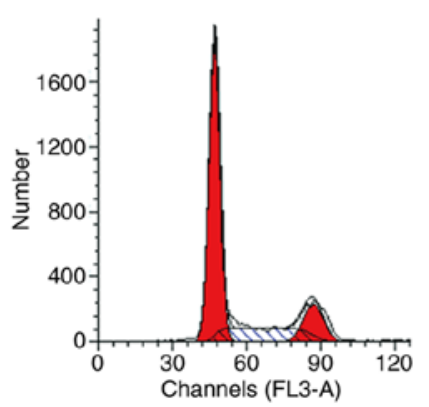

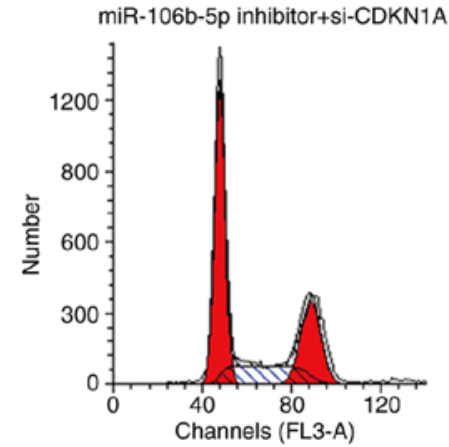

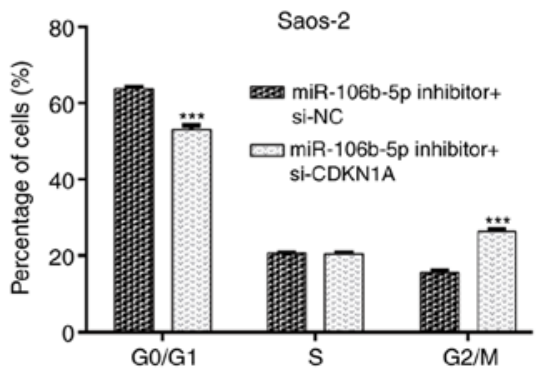

Figure 5. CDKN1A knockdown abolishes the suppressive effects of miR-106b-5p inhibitor transfection on OS cells. (A) Saos-2 cells were co-transfected with miR-106b-5p inhibitor and si-CDKN1A then reverse transcription-quantitative PCR and (B) western blot analysis was used to examine the expression levels of CDKN1A in Saos-2 cells. (C) MTT assay was used to analyze cell proliferation and (D) flow cytometry with propidium iodide staining was used to detect cell cycle distribution. ${ }^{* *} \mathrm{P}<0.01$ and ${ }^{* * *} \mathrm{P}<0.001$ vs. miR-106b-5pinhibitor + si-NC group. CDKN1A, cyclin-dependent kinase inhibitor 1A; miR, microRNA; OS, osteosarcoma; si, small interfering; $\mathrm{NC}$, negative control; OD, optical density.

Furthermore, miR-106b-5p inhibition resulted in reduction of cell proliferation, suggesting that miR-106a-5p could act as oncomiR in OS cell growth.

Uncontrolled cell proliferation is a pathological manifestation of cancer (29). Beneath the complexity of cancer cell proliferation lies a critical event involving defects in cell cycle progression, that have propelled the tumor cells and its progeny into uncontrolled mitotic division (29). The present study investigated the cell-cycle profile to better understand the modulation of OS cell proliferation by miR-106b-5p. 
Knockdown of miR-106b-5p caused a significant increase in the percentage of cells in G0/G1 phase and a decrease in the number of cells in $\mathrm{S}$ and $\mathrm{G} 2 / \mathrm{M}$ phases, suggesting that depletion of miR-106b-5p prevented cell-cycle progression by arresting cells at the G0/G1 phase.

A number of miR-106b-5p target genes have been identified, including SET domain containing 2, histone lysine methyltransferase (11), cathepsin A (27), PTEN (14) and BTG anti-proliferation factor 3 (15), which have pivotal roles in anti-oncogenic processes. Since miRNAs depress the expression of their target mRNAs, the present study hypothesized that miR-106b-5p may target certain genes that function as tumor suppressors. Using bioinformatic prediction and a luciferase reporter assay, it was identified and validated that a cell cycle related gene, CDKN1A, was a direct target gene of miR-106b-5p. A negative correlation between miR-106b-5p and CDKN1A was confirmed by RT-qPCR and western blot analysis. In mammalians, cell cycle progression is partly controlled by a catalytic subunit CDK and its essential activation partner, cyclin (30). Cyclin-dependent kinase inhibitors are known to exert effects by binding to CDK monomers or CDK/cyclin complexes (31). As a universal inhibitor (especially the CDK4/6-cyclin D complexes in G1 phase), CDKN1A serves a significant role in a p53-dependent and independent manner, leading to G0/G1 extension and suppression of further cell proliferation (32).

Transfection experiments were performed to validate that whether CDKN1A was implicated in the miR-106b-5p knockdown-induced reduction of OS cell proliferation. Upregulation of CDKN1A expression mimicked, while CDKN1A knockdown reversed the suppressive effects of miR-106b-5p inhibitor transfection on OS cell proliferation and cell cycle progression. Similarly, miR-106-5p targeting CDKN1A has been reported in gastric cancer (33) and renal cell carcinoma (34). The present study hypothesized that knockdown of miR-106b-3p inhibited proliferation via G0/G1 cell cycle arrest by negatively regulating CDKN1A. However, the present study had a number of limitations including i) lack of immunohistochemical staining of CDKN1A in OS clinical specimens and no investigation into its relationship with miR-106b-5p; ii) the association between miR-106-5p and the clinicopathological features in The Cancer Genome Atlas was not investigated and iii) the effects of miR-106b-5p on cell apoptosis, migration and invasion still need to be explored.

In conclusion, the present study provided strong evidence that miR-106b-5p acts as an oncogene to attenuate the tumor suppressor CDKN1A. Characterization of the miR-106b-5p/CDKN1A functional axis correlation will deepen our understanding of OS etiology and data also indicate the potential of miR-106-5p/CDKN1A axis as a therapeutic target in the treatment of OS.

\section{Acknowledgements}

Not applicable.

\section{Funding}

No funding was received.

\section{Availability of data and materials}

All data generated or analyzed during this study are included in this published article.

\section{Authors' contributions}

QP designed the study. CH and HC performed reverse transcription-quantitative PCR analysis, MTT assay, luciferase reporter assay and western blotting. YL and XL conducted the other functional experiments. CZ collected and analyzed the data. QQ performed the statistical analysis, researched the literature and contributed the manuscript editing. All authors have read and approved the final manuscript.

\section{Ethics approval and consent to participate}

A signed written informed consent was obtained from each patient and the experimental procedures were all in accordance with the guideline of the Ethics Committee of Jingzhou Traditional Chinese Medicine Hospital. The present study was approved by the Ethics Committee of Jingzhou Traditional Chinese Medicine Hospital (grant no. ZA3029C; Jingzhou, China).

\section{Patient consent for publication}

Not applicable.

\section{Competing interests}

The authors declare they have no competing interests.

\section{References}

1. Verrecchia F and Rédini F: Transforming growth factor- $\beta$ signaling plays a pivotal role in the interplay between osteosarcoma cells and their microenvironment. Front Oncol 8: 133, 2018.

2. Luetke A, Meyers PA, Lewis I and Juergens H: Osteosarcoma treatment-Where do we stand? A state of the art review. Cancer Treat Rev 40: 523-532, 2014.

3. Piletic K and Kunej T: MicroRNA epigenetic signatures in human disease. Arch Toxicol 90: 2405-2419, 2016.

4. Apprey V, Wang S, Tang W, Kittles RA, Southerland WM, Ittmann $M$ and Kwabi-Addo B: Association of Genetic ancestry with DNA methylation changes in prostate cancer disparity. Anticancer Res 39: 5861-5866, 2019.

5. Lin S and Gregory RI: MicroRNA biogenesis pathways in cancer. Nat Rev Cancer 15: 321-333, 2015.

6. Wang J, Liu S, Shi J, Li J, Wang S, Liu H, Zhao S, Duan K, Pan X and Yi Z: The Role of miRNA in the diagnosis, prognosis, and treatment of osteosarcoma. Cancer Biother Radiopharm 34: 605-613, 2019.

7. Ma X, Li D, Gao Y and Liu C: miR-451a inhibits the growth and invasion of osteosarcoma via targeting TRIM66. Technol Cancer Res Treat 18: 1533033819870209, 2019.

8. Marx V: Meet some code-breakers of noncoding RNAs. Nat Methods 15: 103-106, 2018.

9. Yamamoto N, Nishikawa R, Chiyomaru T, Goto Y, Fukumoto I, Usui H, Mitsuhashi A, Enokida H, Nakagawa M, Shozu M and Seki N: The tumor-suppressive microRNA-1/133a cluster targets PDE7A and inhibits cancer cell migration and invasion in endometrial cancer. Int J Oncol 47: 325-334, 2015.

10. Wong N and Wang X: miRDB: An online resource for microRNA target prediction and functional annotations. Nucleic Acids Res 43: D146-D152, 2015.

11. Xiang W, He J, Huang $\mathrm{C}$, Chen $\mathrm{L}$, Tao D, Wu X, Wang $\mathrm{M}$, Luo G, Xiao X, Zeng F and Jiang G: miR-106b-5p targets tumor suppressor gene SETD2 to inactive its function in clear cell renal cell carcinoma. Oncotarget 6: 4066-4079, 2015. 
12. Shi DM, Bian XY, Qin CD and Wu WZ: miR-106b-5p promotes stem cell-like properties of hepatocellular carcinoma cells by targeting PTEN via PI3K/Akt pathway. Onco Targets Ther 11: $571-585,2018$.

13. Liu F, Gong J, Huang W, Wang Z, Wang M, Yang J, Wu C, Wu Z and Han B: MicroRNA-106b-5p boosts glioma tumorigensis by targeting multiple tumor suppressor genes. Oncogene 33: 4813-4822, 2014.

14. Chen X, Chen P, Chen SS, Ma T, Shi G, Zhou Y, Li J and Sheng L: miR106b5p promotes cell cycle progression of malignant melanoma by targeting PTEN. Oncol Rep 39: 331-337, 2018.

15. Wei K, Pan C, Yao G, Liu B, Ma T, Xia Y, Jiang W, Chen L and Chen Y: MiR-106b-5p promotes proliferation and inhibits apoptosis by regulating BTG3 in Non-small cell lung cancer. Cell Physiol Biochem 44: 1545-1558, 2017.

16. Malumbres M: Cyclin-dependent kinases. Genome Biol 15: 122, 2014.

17. Kreis NN, Louwen F and Yuan J: Less understood issues: p21(Cip1) in mitosis and its therapeutic potential. Oncogene 34: $1758-1767,2015$.

18. Abbas T and Dutta A: p21 in cancer: Intricate networks and multiple activities. Nat Rev Cancer 9: 400-414, 2009.

19. Fitzgerald AL, Osman AA, Xie TX, Patel A, Skinner H, Sandulache V and Myers JN: Reactive oxygen species and $\mathrm{p} 21 \mathrm{Waf} 1 / \mathrm{Cip} 1$ are both essential for $\mathrm{p} 53$-mediated senescence of head and neck cancer cells. Cell Death Dis 6: e1678, 2015.

20. Wu Z, Liu K, Wang Y, Xu Z, Meng J and Gu S: Upregulation of microRNA-96 and its oncogenic functions by targeting CDKN1A in bladder cancer. Cancer Cell Int 15: 107, 2015.

21. Ohta K, Hoshino H, Wang J, Ono S, Iida Y, Hata K, Huang SK, Colquhoun $S$ and Hoon DS: MicroRNA-93 activates c-Met/PI3K/Akt pathway activity in hepatocellular carcinoma by directly inhibiting PTEN and CDKN1A. Oncotarget 6 : 3211-3224, 2015.

22. Brock M, Haider TJ, Vogel J, Gassmann M, Speich R, Trenkmann M, Ulrich S, Kohler $M$ and Huber LC: The hypoxia-induced microRNA-130a controls pulmonary smooth muscle cell proliferation by directly targeting CDKN1A. Int J Biochem Cell Biol 61: 129-137, 2015.

23. FornariF,MilazzoM,ChiecoP,NegriniM,MarascoE,CapranicoG, Mantovani V, Marinello J, Sabbioni S, Callegari E, et al: In hepatocellular carcinoma miR-519d is up-regulated by p53 and DNA hypomethylation and targets CDKN1A/p21, PTEN, AKT3 and TIMP2. J Pathol 227: 275-285, 2012.
24. Shao M, Geng Y, Lu P, Xi Y, Wei S, Wang L, Fan Q and Ma W: miR-4295 promotes cell proliferation and invasion in anaplastic thyroid carcinoma via CDKN1A. Biochem Biophys Res Commun 464: 1309-1313, 2015.

25. Zhao X, Yang Y, Xu J, Luo Y, Xin Y and Wang Y: Downregulation of microRNA-95-3p suppresses cell growth of osteosarcoma via CDKN1A/p21 expression. Oncol Rep 39: 289-297, 2018.

26. Livak K and Schmittgen T: Analysis of relative gene expression data using real-time quantitative PCR and the 2(-Delta Delta C(T)) method. Methods 25: 402-408, 2000.

27. Ni S, Weng W, Xu M, Wang Q, Tan C, Sun H, Wang L, Huang D, Du X and Sheng W: miR-106b-5p inhibits the invasion and metastasis of colorectal cancer by targeting CTSA. Onco Targets Ther 11: 3835-3845, 2018

28. Lee E, Collazo-Lorduy A, Castillo-Martin M, Gong Y, Wang L, Oh WK, Galsky MD, Cordon-Cardo C and Zhu J: Identification of microR-106b as a prognostic biomarker of p53-like bladder cancers by ActMiR. Oncogene 37: 5858-5872, 2018.

29. Evan GI and Vousden KH: Proliferation, cell cycle and apoptosis in cancer. Nature 411: 342-348, 2001.

30. Wood DJ and Endicott JA: Structural insights into the functional diversity of the CDK-cyclin family. Open Biol 8: 180112, 2018.

31. Sánchez-Martínez C, Gelbert LM, Lallena MJ and de Dios A: Cyclin dependent kinase (CDK) inhibitors as anticancer drugs. Bioorg Med Chem Lett 25: 3420-3435, 2015.

32. Abbadie C, Pluquet O and Pourtier A: Epithelial cell senescence: An adaptive response to pre-carcinogenic stresses? Cell Mol Life Sci 74: 4471-4509, 2017.

33. Dong X, Hu X, Chen J, Hu D and Chen LF: BRD4 regulates cellular senescence in gastric cancer cells via E2F/miR-106b/p21 axis. Cell Death Dis 9: 203, 2018.

34. Sun K, Jia Z, Duan R, Yan Z, Jin Z, Yan L, Li Q and Yang J: Long non-coding RNA XIST regulates miR-106b-5p/P21 axis to suppress tumor progression in renal cell carcinoma. Biochem Biophys Res Commun 510: 416-420, 2019.

(i) (8) This work is licensed under a Creative Commons cc) $\mathrm{EY}$ NO NO Attribution-NonCommercial-NoDerivatives 4.0 International (CC BY-NC-ND 4.0) License. 